\title{
Follow-up of vestibular function in bilateral vestibulopathy
}

\author{
V C Zingler, ${ }^{1}$ E Weintz, ${ }^{1} \mathrm{~K}$ Jahn, ${ }^{1}$ A Mike, ${ }^{2}$ D Huppert, ${ }^{1} \mathrm{~N}$ Rettinger, ${ }^{1} \mathrm{~T}$ Brandt, ${ }^{1}$ \\ M Strupp ${ }^{1}$
}

${ }^{1}$ Department of Neurology, Ludwig-Maximilians University, Klinikum Grosshadern, Munich, Germany; ${ }^{2}$ Department of Neurology, University of Pécs, Hungary

Correspondence to: Dr V C Zingler, Department of Neurology, Ludwig-Maximilians University, Klinikum Grosshadern, Marchioninistraße 15, D-81377 Munich, Germany; vera-carina.zingler@ med.uni-muenchen.de

Received 17 April 2007 Revised 8 June 2007 Accepted 11 June 2007 Published Online First 17 July 2007
ABSTRAC

Objective: Bilateral vestibulopathy (BV) leads to a bilateral deficit of the vestibulo-ocular reflex and has various aetiologies. The main goal of this study was to determine the frequency and degree of recovery or worsening of vestibular function over time.

Methods: 82 patients (59 males, 23 females; mean age at the time of diagnosis 56.3 (SD 17.6) years) were reexamined 51 (36) months after the first examination. All patients underwent a standardised neuro-ophthalmological and neuro-otological examination. Electronystagmography with bithermal caloric irrigation was analysed by measurement of the mean peak slow phase velocity (SPV) of the induced nystagmus. Patients evaluated the course of their disease in terms of balance, gait unsteadiness and health related quality of life.

Results: Statistical analysis of the mean peak SPV of caloric induced nystagmus revealed a non-significant worsening over time (initial mean peak SPV $3.0(3.5)^{\circ} \%$ vs $2.1(2.8)^{\%}$ s). With respect to subgroups of aetiology, only patients with BV due to meningitis exhibited an increasing, but non-significant SPV $(1.0(1.4) \%$ s vs 1.9 $\left.(1.6)^{\circ} / \mathrm{s}\right)$. Vestibular outcome was independent of age, gender, time course of manifestation and severity of BV. Single analysis of all patients showed that a substantial improvement $\geqslant 5 \%$ sccurred in two patients on both sides (idiopathic $n=1$, Sjögren's syndrome $n=1$ ) and in eight patients on one side (idiopathic $n=6$, meningitis $n=1$, Menière's disease $n=1)$. In $84 \%$ of patients there was impairment of their health related quality of life $142 \%$ slight, 24\% moderate, 18\% severe). Forty-three per cent of patients rated the course of their disease as stable, $28 \%$ as worsened and 29\% as improved.

Conclusions: Our data support the view that more than $80 \%$ of patients with BV do not improve. Thus the prognosis of $\mathrm{BV}$ is less favourable than assumed.

Bilateral vestibulopathy (BV) is characterised by impaired or lost function of both peripheral labyrinths or of the eighth nerves. The most frequent complaint of patients with BV is unsteadiness of gait with worsening in darkness and on uneven ground due to deficient vestibulospinal function. Some patients complain of oscillopsia (ie, apparent motion of the visual scene during head movements or when walking). Oscillopsia is caused by involuntary retinal slip as a result of an insufficient vestibulo-ocular reflex (VOR). Furthermore, BV can also lead to impaired spatial memory and navigation associated with hippocampal atrophy. ${ }^{1}$ The diagnosis of $\mathrm{BV}$ is based on the head thrust test, ${ }^{2}{ }^{3}$ a simple bedside test for high frequency VOR function, and bithermal caloric testing with oculographic recordings to test for a low frequency VOR deficit. BV is a rare vestibular disorder of various aetiologies (eg, ototoxic drugs, meningitis or Menière's disease).4Despite intensive examinations, the aetiology remains unclear in approximately $50 \%{ }^{6}$

Little is known about the course of the disease, especially the recovery. A few small case series have reported contradictory recovery rates. ${ }^{5-9}$ In a report on 11 patients with idiopathic BV (eight patients with simultaneous and three patients with sequential onset of BV), partial recovery was detected in four patients with simultaneous and in all three patients with sequential BV after a follow-up of 1-7 years. Complete recovery did not occur in any of the patients. ${ }^{5}$ Improvement of vestibular function was also described in single cases of different aetiologies, ${ }^{9}$ in particular when BV was caused by serous rather than suppurative destructive labyrinthitis. ${ }^{41011}$ In contrast, in a 5 year follow-up study ${ }^{7}$ of seven patients with $\mathrm{BV}$, Baloh et al did not find significant improvement in vestibular excitability. Furthermore, in a recent study, the effectiveness of immunosuppressive treatment of patients with BV and inner ear antibodies was moderate in four of 12 patients and only transient in two of them. ${ }^{8}$ Based on our clinical experience, the prognosis of $\mathrm{BV}$ is less favourable than hitherto reported. Therefore, we examined 82 patients with BV of various aetiologies and clinically re-evaluated them after a mean follow-up time of 51 (36) months (range 3 months to 13 years). The initial diagnosis of $\mathrm{BV}$ and clinical re-evaluation were made according to a standardised protocol, including a neuro-ophthalmological and neuro-otological workup. We not only determined the frequency and extent of recovery or worsening of vestibular function and their dependence on the aetiology of $\mathrm{BV}$, but also the impairment of quality of life.

\section{PATIENTS AND METHODS}

The diagnosis of BV was based on the following criteria: (1) a pathological head thrust test, ${ }^{2}{ }^{3}$ which indicates a high frequency deficit of the VOR and/ or (2) bilaterally diminished or absent caloric responses on an electronystagmogram (ENG), indicating a low frequency deficit. ${ }^{4}{ }^{12}$

Complete BV was characterised by pathological head thrust tests and the absence of caloric responses on both sides.

Incomplete $\mathrm{BV}$ was characterized by one of the following responses:

- bilateral pathological head thrust tests and a calorically elicited nystagmus with a mean 
peak slow phase velocity (SPV) of $<5 \%$ for each caloric irrigation on both sides;

- bilateral pathological head thrust tests and caloric responses $>5 \%$ on one or both sides; or

- normal head thrust tests and loss of bilateral responses or reduced responses $\left(<5^{\circ} / \mathrm{s}\right)$ to caloric irrigation on both sides.

\section{Definition of aetiology}

Patients were classified as having either a "definite" or "probable" cause of BV. This classification was based on the previously published literature on BV. ${ }^{4}$ The aetiologies were carefully reassessed by the senior authors.

A total of 163 patients who had been diagnosed with BV in the Department of Neurology, University of Munich, between 1993 and 2005 were contacted. Ninety-five patients responded and 82 finally came to the follow-up examinations. The current address could not be determined for 37 patients, 28 patients did not respond and three had died in the meantime.

A standardised detailed history was obtained from all patients at the first and follow-up examinations. The following parameters were assessed: (1) recurrent (preceding or current) attacks of vertigo or dizziness: onset, duration, time course, frequency and associated symptoms (oscillopsia, double vision, blurred vision, loss of sight, phonophobia, photophobia, headache, nausea, vomiting); (2) unsteadiness of gait, in particular in the dark or on uneven ground; (3) oscillopsia during head movements or during locomotion; (4) recurrent or sustained ear symptoms (tinnitus, hearing loss, fullness of the ear); (5) past medical history with regard to migraine, polyneuropathy, exposure to toxins, antibiotics or diuretics, cardiovascular risk factors (eg, arterial hypertension, diabetes mellitus) or other neurological, ophthalmological, ENT or internal diseases (eg, autoimmune disorders, cancer); (6) family history of impaired audiovestibular function.

All patients underwent a complete neurological, neuroophthalmological and neuro-otological examination. The neuro-ophthalmological and neuro-otological examination included the head thrust test, evaluation for spontaneous nystagmus with Frenzel's goggles, gaze evoked nystagmus, smooth pursuit, saccades, optokinetic nystagmus, visual fixation suppression of the VOR, rebound nystagmus and head shaking nystagmus (for general neuro-ophthalmological and neuro-otological testing, see Brandt and Strupp ${ }^{13}$ ). A cerebellar ocular motor disorder was characterised by either one or a combination of the following ocular motor dysfunctions: rebound nystagmus, downbeat nystagmus, pathological saccades and pathological visual fixation suppression of the VOR.

ENG with bithermal caloric testing $\left(30^{\circ} \mathrm{C}\right.$ and $\left.44^{\circ} \mathrm{C}\right)$ was performed in all patients to determine mean peak SPV using Igor Pro Wave Metric software (V.3.13). We determined the mean peak SPV of the 10 fastest slow phases for each side and temperature. For technical reasons, three ENGs performed at follow-up could not be evaluated; these patients were therefore excluded. None of the patients was on medications with a relevant influence on vestibular testing, such as benzodiazepines, analgesics or anticonvulsants.

A cranial MRI was performed in 78 patients. Specific laboratory tests were initiated according to the suspected specific aetiologies (autoantibodies against inner ear structures, vitamin B12 and folate, antinuclear antibodies, complement factors C3 and C4 and CSF test). Autoantibodies against inner ear structures were determined as previously reported by our group. ${ }^{14}$ All patients performed vestibular exercises and balance training using a video or DVD for at least 3 months. ${ }^{15}$

\section{Subjective parameters}

The patients were asked: (1) to evaluate the impairment of their health related quality of life measured at the time of the second evaluation with a self-classification system (with respect to mobility, self-care and daily activities) consisting of four degrees of severity in comparison with their situation before manifestation of BV (not impaired and slightly, moderately or severely impaired); (2) to evaluate the course of their disease by comparing the change in balance and gait unsteadiness (unchanged, worse, improved); (3) if they had noticed changes or difficulties in spatial navigation.

\section{Statistical analysis}

The mean peak SPV of each patient was calculated as follows: peak SPV $(\%)$ after caloric testing at $30^{\circ} \mathrm{C}$ and $44^{\circ} \mathrm{C}$ for the right and left sides were added up, and the sum was divided by 4. For statistical analysis, a main effects ANOVA (Statistica 6.0,

Table 1 Number of patients (\%) in subgroups with different aetiologies and types of manifestation of bilateral vestibulopathy* and number of patients with bilateral pathological head thrust tests and electronystagmogram at the initial and follow-up examinations

\begin{tabular}{|c|c|c|c|c|c|}
\hline & \multirow{2}{*}{$\begin{array}{l}\text { Patients } \\
\text { (n (\%)) }\end{array}$} & \multicolumn{2}{|c|}{ ENG (mean (SD) peak SPV) } & \multicolumn{2}{|c|}{$\begin{array}{l}\text { Bilateral pathological head } \\
\text { thrust tests }\end{array}$} \\
\hline & & Initial & Follow-up & Initial (n) & Follow-up (n) \\
\hline Aetiology of BV & $79(100)$ & $3.0(3.5)$ & $2.1(2.8)$ & 72 & 72 \\
\hline Antibiotics & $10(13)$ & $2.3(2.2)$ & $0.8(1.1)$ & 8 & 9 \\
\hline Meningitis & 7 (9) & $1.0(1.4)$ & $1.9(1.6)$ & 7 & 6 \\
\hline Pathological inner ear antibodies & 7 (9) & $1.8(2.0)$ & $1.6(2.0)$ & 7 & 7 \\
\hline Menière's disease & $6(8)$ & $2.5(3.0)$ & $1.9(3.2)$ & 6 & 5 \\
\hline Miscellaneous: & $10(13)$ & $6.6(4.8) \ddagger$ & $4.4(5.6)$ & 9 & 9 \\
\hline Idiopathicf: & $39(49)$ & $4.9(6.1) \dagger$ & $3.9(5.4)$ & 35 & 36 \\
\hline Sequential BV & $30(38)$ & $3.0(3.5)$ & $2.1(2.8)$ & 28 & 30 \\
\hline Slowly progressive BV & $49(62)$ & $4.6(5.8)$ & $3.6(5.4)$ & 47 & 45 \\
\hline
\end{tabular}

${ }^{*}$ Some of the percentages have been rounded up, others rounded down.

$\dagger$ The mean peak SPV of each patient was calculated as follows: all peak SPVs after caloric testing at $30^{\circ} \mathrm{C}$ and $44^{\circ} \mathrm{C}$ for the right and left sides were added, and the sum was divided by 4 .

\$Only those patients were included who showed either a mean peak SPV $\leqslant 5 \%$ and/or had a bilateral pathological head thrust test.

The total number of all patients was 79 .

BV, bilateral vestibulopathy; ENG, electronystagmogram; SPV, slow phase velocity. 
Statsoft) was performed with mean peak slow phase velocities of caloric responses at the two time points as dependent variables and the following categorical predictors: "aetiology" (see table 1, six levels), "course" (progressive vs sequential, two levels), "gender" (two levels), "age" (patients grouped by decades, eight levels) and "completeness" (complete vs incomplete BV, two levels). Significance was defined as an alpha level of 0.05 . Data in the figure are given as mean $(95 \%$ confidence intervals).

\section{RESULTS}

\section{Study population}

Eighty-two patients with a mean age of 56.3 (SD 17.6) years at the time of diagnosis were included. The diagnosis was made, on average, 71 (119) months (median 36) after the onset of the first symptoms. The majority were male $(72 \%, n=59$, mean age 55.8 (15.2) years; females 28\%, $n=23$, mean age 62.9 (15.0) years). Thirty-two per cent of all patients fulfilled the
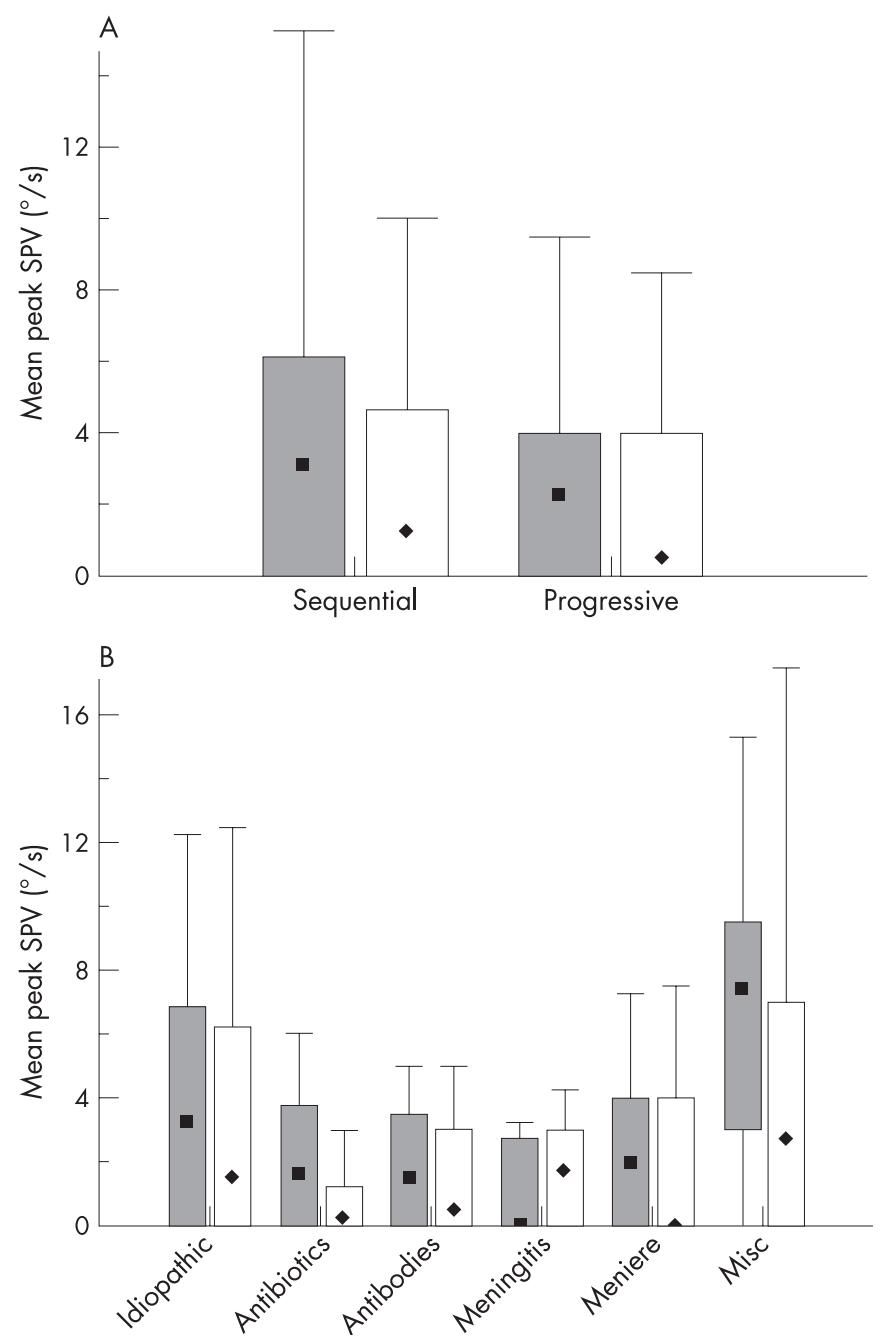

Figure 1 (A, B) Response to caloric irrigation in the initial and follow-up electronystagmogram (mean (SD) peak slow phase velocity (SPV)) in patients with sequential and slowly progressive bilateral vestibulopathy (BV) (A) and for subgroups with BV of various aetiologies (B). Filled column, first examination; open column, follow-up examination. Misc, miscellaneous; antibodies, pathological inner ear antibodies. Symbols in the columns (square, diamond) = median; horizontal lines of the boxes $=25$ th and 75 th percentiles; whiskers above and below the boxes $=$ minimum and maximum values. above listed criteria for complete BV $(\mathrm{n}=26)$ and the remaining $(n=56)$ for incomplete BV. In the latter, a subgroup $(n=5)$ with bilaterally normal head thrust tests, but pathological caloric responses, was identified.

The ENG of three patients could not be evaluated for technical reasons and thus the following statistical analyses refer to the remaining 79 patients.

\section{Time course of the disease manifestation}

Episodes of vertigo or dizziness preceded the manifestation of BV in $38 \%$ of patients $(n=30)$ who had a so-called sequential course. BV developed simultaneously, most often slowly progressively in $62 \%$ of all patients $(n=49)$, as indicated by a patient history of progressive unsteadiness of gait and oscillopsia.

\section{Aetiology of BV}

The definite or probable cause was defined in $51 \%$ of all patients $(n=40)$. The most frequent causes were adverse effects of aminoglycosides ( $13 \%$ of total, $\mathrm{n}=10)$ : gentamycin only in six (of 10) patients, gentamycin plus other antibiotics (streptomycin, vancomycin, penicillin) in three (of 10) patients and gentamycin plus furosemide in one (of 10) patients. Nine per cent of patients $(n=7)$ had suffered from meningoencephalitis and another $9 \%(n=7)$ had autoantibodies against inner ear structures. Another group ( $8 \%$ of the total, $n=6$ ) consisted of patients with Menière's disease. There was also a group (13\% of the total, $\mathrm{n}=10$ ) with "miscellaneous" causes: side effects caused by high dose therapy with furosemide $(n=1)$, otosclerosis $(n=1)$, vitamin B12 deficiency $(n=2)$, alcoholism $(n=2)$, positive family history for inner ear disease $(n=1)$, an association of BV with spinocerebellar ataxia type $6(n=1)$ or systemic autoimmune diseases (Sjögren's syndrome and ulcerative colitis) $(n=2)$. The underlying aetiology of BV remained unclear in $49 \%$ (so-called idiopathic BV; $\mathrm{n}=39$ ).

\section{BV and its association with cerebellar symptoms}

Ten of all included patients with BV (nine males, one female) were also diagnosed as having a cerebellar syndrome at the first examination. The following cerebellar signs were found: cerebellar ocular motor disorders in all 10 patients, ataxia of the limbs in nine patients and dysarthrophonia in four patients. None of the other included patients with BV developed a cerebellar syndrome in the period between the first and the second examinations. BV was caused by spinocerebellar ataxia

Table 2 Subjective evaluation of the course of the disease, health related quality of life and spatial navigation at the time of follow-up

\begin{tabular}{ll}
\hline Subjective evaluation & $\begin{array}{l}\text { Patients } \\
\text { (n (\%)) }\end{array}$ \\
\hline Quality of life & \\
Not impaired & $13(16)$ \\
Slightly impaired & $33(42)$ \\
Moderately impaired & $19(24)$ \\
$\quad$ Severely impaired & $14(18)$ \\
Spatial orientation & \\
Not impaired & $69(87)$ \\
Impaired & $10(13)$ \\
Disease course & \\
$\quad$ Unchanged & $34(43)$ \\
$\quad$ Worse & $22(28)$ \\
Improved & $23(29)$ \\
\hline
\end{tabular}


Table 3 Subjective evaluation of the course of the disease

\begin{tabular}{lccl}
\hline Aetiology & $\begin{array}{l}\text { Worse } \\
\text { (n (\%)) }\end{array}$ & $\begin{array}{l}\text { Unchanged } \\
\text { (n (\%)) }\end{array}$ & $\begin{array}{l}\text { Improved } \\
\text { (n (\%)) }\end{array}$ \\
\hline Idiopathic & $14(36)$ & $17(44)$ & $8(21)$ \\
Antibiotics & $3(30)$ & $4(40)$ & $3(30)$ \\
Pathological inner ear antibodies & $1(14)$ & $4(57)$ & $2(29)$ \\
Meningitis & $3(43)$ & $3(43)$ & $1(14)$ \\
Menière's disease & $1(17)$ & $1(17)$ & $4(67)$ \\
Miscellaneous & $1(10)$ & $5(50)$ & $4(40)$ \\
\hline
\end{tabular}

The results are given for different subgroups (some of the percentages have been rounded up, others rounded down).

$(\mathrm{n}=1)$ and alcoholism $(\mathrm{n}=2)$. The other patients had idiopathic BV.

\section{Cranial imaging}

MRI studies were performed in 78 of all included 82 patients. The following abnormalities were found: cerebellar atrophy $(n=6)$, other cerebellar lesions (eg, cerebellar infarction, $n=2)$, unilateral acoustic neuroma $(n=1)$, generalised cerebral atrophy $(n=4)$ and supra- or infratentorial vascular lesions $(n=10)$.

\section{Follow-up with re-evaluation of vestibular function}

The time between the initial diagnosis and follow-up examination was 51 (36) months (range 3 months to 13 years; median 41 months). The initial ENG showed no significant differences in response to caloric irrigation with respect to the aetiology subgroups or time course of manifestation. Table 1 shows the mean peak SPV after caloric irrigation and the number of patients with bilateral pathological head thrust tests at the first and follow-up examinations. The results are also given for different aetiologies and time courses of manifestation. In general, the mean peak SPV after caloric irrigation tended to decrease at follow-up, but not significantly.

This was true for the total study population as well as for patients with different time courses of manifestation of BV (fig 1A) and aetiologies (fig 1B). Only patients with BV due to meningitis had slightly increased mean peak SPVs at the followup investigation $(n=7)$. Single analysis of all patients showed that a substantial improvement in caloric responses $\geqslant 5 \% \mathrm{~s}$ occurred in two patients on both sides (idiopathic $\mathrm{n}=1$, Sjögren's syndrome $\mathrm{n}=1$ ) and in eight patients on one side (idiopathic $n=6$, meningitis $n=1$, Menière's disease $n=1$ ). The patient with Sjögren's syndrome had received immunosuppressive treatment with cortisone and azathioprine. The statistical analysis of vestibular outcome with regard to age, gender and complete versus incomplete BV also revealed no significant differences. Oscillopsia during head and body movements were reported by $56 \%$ of all patients $(n=44)$ at the first examination and by $53 \%(n=42)$ at follow-up. Gait unsteadiness was reported by all patients at both examinations.

\section{Subjective evaluation of the course of the disease, quality of life and spatial navigation}

Table 2 gives an overview of how patients subjectively evaluated the course of their disease, their health related quality of life and their spatial navigation. Most of the patients (43\%) considered the course of their disease stable. The rest estimated their clinical condition as either worsened (29\%) or improved (28\%).

As regards the different aetiology subgroups (table 3), only four of six patients with Menière's disease felt that they had improved at follow-up. These patients had been treated with high doses of betahistine dihydrochloride (48 $\mathrm{mg}$ three times daily). At follow-up, attacks of vertigo had completely stopped in three of these patients, and the duration and frequency of attacks were markedly decreased in one patient. The majority of patients (42\%) stated that BV caused a slight impairment of their health related quality of life (24\% moderate and 18\% severe impairment). Only a minority of patients (16\%) felt that BV had not impaired their quality of life. Thirteen per cent of patients had noticed an impaired spatial navigation.

\section{DISCUSSION}

The major finding of this follow-up study in patients with BV was that, in general, vestibular function remained unchanged with a non-significant tendency of slow worsening. The vestibular outcome was independent of the underlying causative factors, type of manifestation, severity of vestibular loss, age and gender. This supports the findings of a longitudinal study ${ }^{7}$ in a small group of seven patients with BV of different aetiologies and 57 healthy subjects who returned for yearly examinations of vestibular function over a period of 5 years. In this study, no improvement was found in BV patients; the normal older subjects (all over the age of 75 years) exhibited an age related decrement in their vestibular responses without any associated symptoms or signs of disequilibrium. It is known from various studies that all structures relevant for vestibular function (ie, the vestibular end organs and central vestibular pathways) degenerate continuously with age. Hair cell loss precedes those seen in the vestibular nerve and Scarpa's ganglion. ${ }^{16-19}$ Thus it can be assumed that the mild decrement in vestibular responses in our study can be attributed to progression of the disease as well as vestibular aging. Because of the study design of our and previous investigations, ${ }^{5-9}$ most of the patients presented with a chronic condition. This implies that those patients with acute onset of BV and early recovery (such as patients in the intensive care unit with BV caused by meningitis or treatment with ototoxic antibiotics) might remain undetected by this approach. The latter argument is supported by the improvement of patients with BV due to meningitis in our study. This is supported by previous case reports. It has been stated that patients with serous rather than suppurative meningitis can exhibit partial recovery. ${ }^{10} 11$

In our study, none of the patients with BV caused by ototoxic antibiotics showed a substantial improvement. However, regeneration of receptor cells and subsequent functional recovery after (gentamycin induced) damage of the auditory and vestibular systems in many vertebrates is well known. ${ }^{20}{ }^{21}$

The subjective evaluation of the course of the disease was similar to the results of our objectively achieved findings for vestibular testing. Most of the patients estimated their clinical condition as being either unchanged or worse. In particular, only a minority of patients reported an improvement in gait unsteadiness. Part of this improvement might be due to vestibular exercises and balance training, which all of our patients had for at least 3 months. The usefulness of vestibular rehabilitation in unilateral vestibular failure ${ }^{15}$ and BV $^{22}{ }^{23}$ has been reported previously. The improvement after vestibular rehabilitation with clear locomotor gains has been described in approximately $50 \%$ of $\mathrm{BV}$ patients who received intensive training over 3-12 months. ${ }^{22}$ Only patients with Menière's disease frequently assessed their course of disease as being favourable. This might be explained by spontaneous or drug induced reduction or cessation of vertigo attacks in the later stage of the disease. 
About two-thirds of our patients rated their health related quality of life as slightly to moderately impaired. Nearly $90 \%$ of patients estimated their spatial navigation as unchanged since the first manifestation of BV. A previous study had shown that complete BV can lead to impaired spatial memory and navigation associated with hippocampal atrophy. ${ }^{1}$

This study has some limitations as to completeness of vestibular testing with respect to otolith (vestibular evoked myogenic potentials) and canal function, dynamic visual acuity, evaluation of hearing impairment, posturography and gait analysis, or the application of a health self-classification system such as the EQ-5D ${ }^{24}$ or specific dizziness rating scales. None of these tests was included because they were not routinely performed at the first examination. We chose the head thrust test and bithermal caloric testing with $30^{\circ} \mathrm{C}$ and $44^{\circ} \mathrm{C}$ (instead of rotational testing) because it is the gold standard for documenting $\mathrm{BV}^{13}$ and allows side specific assessment of (residual) vestibular function. One may argue that in patients who already have minimal to no caloric responses to start with, it is not surprising that no statistical difference was seen at follow-up. Caloric testing with ice water would have been an additional tool to detect slight improvement. However, the main goal of this study was to investigate a relevant improvement or worsening of vestibular function over time. In addition, according to our experience with many patients, caloric testing with ice water is not well tolerated and was therefore not routinely used in our patients.

In conclusion, our data support the view that more than $80 \%$ of patients with BV do not improve. Thus the prognosis of BV is less favourable than assumed. This emphasises the need for intensive vestibular rehabilitation ${ }^{25}$ to reduce the burden of disease.

Acknowledgements: We thank Ms Judy Benson for copyediting the manuscript. Competing interests: None.

Ethics approval: Ethics approval was obtained.

\section{REFERENCES}

1. Brandt T, Schautzer F, Hamilton DA, et al. Vestibular loss causes hippocampal atrophy and impaired spatial memory in humans. Brain 2005;128:2732-41.
2. Halmagyi GM, Curthoys IS. A clinical sign of canal paresis. Arch Neurol 1988; 45:737-9.

3. Jorns-Häderli M, Straumann D, Palla A. Accuracy of the bedside head impulse test in detecting vestibular hypofunction. J Neurol Neurosurg Psychiatry 2007;78:111318.

4. Rinne T, Bronstein AM, Rudge $P$, et al. Bilateral loss of vestibular function: clinical findings in 53 patients. J Neurol 1998;245:314-21.

5. Vibert D, Liard P, Häusler R. Bilateral idiopathic loss of peripheral vestibular function with normal hearing. Acta Otolaryngol 1995;115:611-15.

6. Zingler VC, Cnyrim C, Jahn K, et al. Causative factors and epidemiology of bilateral vestibulopathy in 255 patients. Ann Neurol 2007:61:524-32.

7. Baloh RW, Enrietto J, Jacobson KM, et al. Age-related changes in vestibular function: a longitudinal study. Ann N Y Acad Sci 2001;942:210-19.

8. Deutschlander A, Glaser M, Strupp M, et al. Immunosuppressive treatment in bilateral vestibulopathy with inner ear antibodies. Acta Otolaryngol 2005;125:848-51.

9. Frese KA, Reker U, Maune S. Der beidseitige Vestibularisausfall. HNO 2003;51:2215.

10. Fortnum HM. Hearing impairment after bacterial meningitis. Arch Dis Child 1982;67:1128-33.

11. Bronstein $\mathbf{A M}$, Morland $\mathrm{AB}$, Ruddock $\mathrm{KH}$, et al. Recovery from bilateral vestibular failure: implications for visual and cervico-ocular function. Acta Otolaryngol Suppl 1995;520:405-7.

12. Baloh RW, Honrubia V, Yee RD, et al. Changes in the human vestibulo-ocular reflex after loss of peripheral sensitivity. Ann Neurol 1984;16:222-8.

13. Brandt T, Strupp M. General vestibular testing. Clin Neurophysiol 2005;116:406-26

14. Arbusow V, Strupp M, Dieterich $M$, et al. Serum antibodies against membraneous labyrinth in patients with "idiopathic" bilateral vestibulopathy. J Neurol 1998;245:132-6.

15. Strupp M, Arbusow V, Maag KP, et al. Vestibular exercises improve central vestibulospinal compensation after vestibular neuritis. Neurology 1998;51:838-44.

16. Bergstrom B. Morphology of the vestibular nerve. II The number of myelinated vestibular nerve fibers in man at various stages. Acta Otolaryngol 1973;76:173-9.

17. Rosenhall U. Degenerative patterns in the ageing human vestibular neuroepithelia. Acta Otolaryngol 1973;76:208-28.

18. Lopez I, Hornrubia V, Baloh RW. Ageing and the human vestibular nucleus. J Vestib Res 1997; 7:77-85.

19. Jahn K, NaeßI A, Schneider E, et al. Inverse U-shaped curve for age dependency of torsional eye movement responses to galvanic vestibular stimulation. Brain 2003;126:1579-89.

20. Zakir M, Dickmann JD. Regeneration of vestibular otolith afferents after ototoxic damage. J Neurosci 2006;26:2881-93.

21. Taura A, Kojima K, Ito J, et al. Recovery of hair cell function after damage induced by gentamicin in organ culture of rat vestibular maculae. Brain Res 2006;1098:3348.

22. Krebs DE, Gill-Body KM, Parker SW, et al. Vestibular rehabilitation: usefull but not universally so. Otolaryngol Head Neck Surg 2003;128:240-50.

23. Gillespie MB, Minor LB. Prognosis in bilateral vestibular hypofunction. Laryngoscope 1999; 109:35-41.

24. EuroQuol Group. Euroquol: a new facility for the measurement of health-related quality of life. Health Policy 1990;16:199-208.

25. Herdmann SJ. Role of vestibular adaptation in vestibular rehabilitation. Otolaryngol Head Neck Surg 1998;119:49-54. 


\section{Follow-up of vestibular function in bilateral vestibulopathy}

V C Zingler, E Weintz, K Jahn, et al.

J Neurol Neurosurg Psychiatry 2008 79: 284-288 originally published online July 17,2007

doi: 10.1136/jnnp.2007.122952

Updated information and services can be found at:

http://jnnp.bmj.com/content/79/3/284.full.html

These include:

References This article cites 25 articles, 6 of which can be accessed free at: http://jnnp.bmj.com/content/79/3/284.full.html\#ref-list-1

Article cited in:

http://jnnp.bmj.com/content/79/3/284.full.html\#related-urls

Email alerting Receive free email alerts when new articles cite this article. Sign up in service the box at the top right corner of the online article.

Topic Articles on similar topics can be found in the following collections Collections

Infection (neurology) (392 articles)

Ophthalmology (669 articles)

Notes

To request permissions go to:

http://group.bmj.com/group/rights-licensing/permissions

To order reprints go to:

http://journals.bmj.com/cgi/reprintform

To subscribe to BMJ go to:

http://group.bmj.com/subscribe/ 